\title{
Upfront osimertinib in EGFR-mutated non-small cell lung cancer: is brain still a sanctuary?
}

\author{
Alessandro Leonetti ${ }^{1,2}$, Francesco Facchinetti ${ }^{3}$, Marcello Tiseo $^{1,4}$ \\ ${ }^{1}$ Medical Oncology Unit, University Hospital of Parma, Parma, Italy; ${ }^{2}$ Department of Medical Oncology, VU University Medical Center, Amsterdam \\ The Netherlands; ${ }^{3}$ INSERM U981, Gustave Roussy Cancer Campus, Université Paris Saclay, Villejuif, France; ${ }^{4}$ Department of Medicine and \\ Surgery, University of Parma, Parma, Italy \\ Correspondence to: Marcello Tiseo, MD, PhD. Department of Medicine and Surgery, University of Parma, Parma, Italy; Medical Oncology Unit, \\ University Hospital of Parma, Via Gramsci, 14, 43126 Parma, Italy. Email: mtiseo@ao.pr.it. \\ Comment on: Reungwetwattana T, Nakagawa K, Cho BC, et al. CNS Response to Osimertinib Versus Standard Epidermal Growth Factor \\ Receptor Tyrosine Kinase Inhibitors in Patients With Untreated EGFR-Mutated Advanced Non-Small-Cell Lung Cancer. J Clin Oncol \\ 2018:JCO2018783118. [Epub ahead of print].
}

Submitted Nov 18, 2018. Accepted for publication Nov 26, 2018.

doi: 10.21037/atm.2018.11.69

View this article at: http://dx.doi.org/10.21037/atm.2018.11.69

In the last 10 years, the prognosis of advanced non-small cell lung cancer (NSCLC) patients harboring epidermal growth factor receptor $(E G F R)$ mutations has dramatically improved, since the introduction of selective EGFRtyrosine kinase inhibitors (TKIs). Median overall survival (OS) of EGFR-mutated NSCLC patients treated frontline with first- or second-generation EGFR-TKIs (gefitinib, erlotinib or afatinib) exceeded 30 months, and nearly $15 \%$ of patients were still alive at 5 years (1). However, as patients live longer, the likelihood of developing brain metastases $(\mathrm{BM})$ during the natural course of the disease broadens, and this event significantly impairs quality of life and affects patients' long-term outcomes (2). This is even more troubling when considering that EGFR-mutant patients have a greater incidence of $\mathrm{BM}(40 \%$ up to $70.3 \%)$ than patients with wild-type $\operatorname{EGFR}(38 \%)(3,4)$. The efficacy of the novel targeted-treatments, which have a proven extra-cranial performance, is thus often limited by the onset of new brain lesions as well as progression of preexisting ones. Indeed, first- and second-generation EGFR-TKI have a relatively low brain penetration potential, which can explain why the central nervous system (CNS) is a frequent site of failure after achieving a meaningful clinical benefit with some TKI $(5,6)$. The acquisition of T790M resistance mutation in extra-cranial localizations in $50-60 \%$ of patients is due to the selective pressure on tumor cells exerted by TKI (7). On the other hand, the blunt effect of first-/second-generation agents on CNS is mainly due to pharmacokinetics limitations, precluding a significant blood-brain penetration, and only to a lower extent to T790M emergence (8). Table 1 gathers data regarding activity and efficacy of EGFR-TKI on CNS disease.

Beyond first- and second-generation TKI, osimertinib, a potent irreversible TKI that targets the classic EGFRactivating mutations as well as the gatekeeper T790M resistance mutation, has been approved for EGFRmutated patients who harbored the T790M mutation and experienced disease progression after treatment with firstor second-generation EGFR TKI (17). Regarding CNS efficacy in this setting, osimertinib showed a robust clinical CNS activity (Table 1), achieving a CNS overall response rate (ORR) that ranged from $54 \%$ (14) up to $70 \%(15)$. When administered in second-line, osimertinib more than doubled the median CNS progression-free survival (PFS) obtained with platinum-based chemotherapy in this setting [11.7 versus 5.6 months, hazard ratio (HR), $0.32 ; 95 \%$ confidence interval (CI), 0.15-0.69; $\mathrm{P}=0.004]$, and CNS responses were also durable (15). Moreover, in the pooled subgroup analysis of the two global phase II trials (AURA extension and AURA2), 6 out of 50 patients (12\%) treated with osimertinib obtained a complete CNS response, and benefit was observed irrespective of prior brain radiotherapy (14).

Moving the third-generation inhibitor in the first-line setting (18), in the phase III FLAURA trial, osimertinib was strongly superior to erlotinib and gefitinib in untreated EGFR-positive patients, achieving a PFS of 18.9 versus 
Table 1 CNS activity of EGFR-TKIs in EGFR-mutated NSCLC

\begin{tabular}{|c|c|c|c|c|c|c|c|}
\hline EGFR-TKI & Reference & Study design & $\begin{array}{c}\mathrm{N} \text { of } \\
\text { patients }\end{array}$ & $\begin{array}{l}\text { Line of } \\
\text { treatment }\end{array}$ & CNS ORR & $\mathrm{CR}$ & $\begin{array}{l}\text { CNS PFS }(95 \% \mathrm{Cl}) \\
\text { (months) }\end{array}$ \\
\hline Erlotinib & Porta et al., Eur Respir J, 2011 (9) & Retrospective & 17 & $\geq 1$ & $82 \%$ & $47 \%$ & $11.7(7.9-15.5)$ \\
\hline \multirow[t]{2}{*}{ Gefitinib } & Hotta et al., Lung Cancer, 2004 (11) & Retrospective & $14^{\#}$ & $\geq 1$ & $43 \%$ & $7 \%$ & NA \\
\hline & luchi et al., Lung Cancer, 2013 (12) & Phase II & $41^{\#}$ & 1 & $88 \%$ & $32 \%$ & $14.5(10.2-18.3)$ \\
\hline Afatinib & $\begin{array}{l}\text { Schuler et al., J Thorac Oncol, } 2016 \\
\text { (13) }\end{array}$ & $\begin{array}{l}\text { Pooled analysis of } \\
\text { LUX-Lung } 3 \text { and } \\
\text { LUX-Lung } 6\end{array}$ & 81 & 1 & NA & NA & $\begin{array}{l}\text { TTP LUX-Lung 3: } \\
15.2 \text { (7.7-29.0); } \\
\text { TTP LUX-Lung 6: } \\
15.2 \text { (3.8-23.7) }\end{array}$ \\
\hline \multirow{2}{*}{ Osimertinib } & Wu et al., J Clin Oncol, 2018 (15) & Phase III & 116 & $2^{\S}$ & $\begin{array}{l}\text { cEFR: } 70 \% \\
\text { cFAS: } 40 \%\end{array}$ & $\begin{array}{l}\text { cEFR: } 7 \% \\
\text { cFAS: } 15 \%\end{array}$ & cFAS: $11.7(10-N C)$ \\
\hline & $\begin{array}{l}\text { Reungwetwattana et al., } \\
J \text { Clin Oncol, } 2018 \text { (16) }\end{array}$ & Phase III & 128 & 1 & $\begin{array}{l}\text { cEFR: } 91 \% \\
\text { cFAS: } 66 \%\end{array}$ & $\begin{array}{l}\text { cEFR: } 23 \% \text {; } \\
\text { cFAS: } 41 \%\end{array}$ & cFAS: NR (16.5-NC) \\
\hline
\end{tabular}

*, number of patients assessed for EGFR-TKI intracranial response; ", Japanese only patients; ${ }^{\text {, }}$, patients with progressed to previous EGFR-TKI treatment due to the onset of T790M mutation. cEFR, CNS evaluable-for-response set; cFAS, CNS full-analysis set; CI, confidence interval; CNS, central nervous system; CR, complete response; CUP, compassionate use program; EGFR-TKI, epidermal growth factor receptor-tyrosine kinase inhibitor; N, number; NA, not available; NC, not calculated; ORR, overall response rate; PFS, progression-free survival; TTP, time to progression.

10.2 months obtained with standard EGFR-TKI (HR, 0.46; 95\% CI, 0.37-0.57; P<0.001) (19). Osimertinib has been recently FDA- (20) and EMA- (21) approved for the first-line treatment of NSCLC driven by conventional EGFR mutations. Of note, the greater brain-blood barrier penetration potential of osimertinib in preclinical models, compared to gefitinib or erlotinib $(22,23)$, had likely contributed to positive FLAURA results, also in terms of signals of OS benefit (19). Nevertheless, to date, a prospective analysis of intracranial efficacy of osimertinib, when administered frontline in EGFR-mutated patients, has been missing.

In the paper by Reungwetwattana and colleagues, the authors reported the final results of a preplanned subgroup analysis of the phase III FLAURA trial specifically aimed to explore the CNS efficacy of osimertinib in the firstline treatment of EGFR-mutated NSCLC patients (16). Among 556 participants of the FLAURA trial, 200 individuals $(36.0 \%)$ had brain radiological exams available at baseline, and 128 of them (64\%) had CNS metastases, both measurable and/or non-measurable by RECIST criteria (16). The number of patients with at least one measurable BM was well balanced between the two arms, and it consisted of $22(8 \%)$ and 19 (7\%) patients for osimertinib and standard TKIs group, respectively. Importantly, the trial allowed the enrollment of patients with known and pre-irradiated $\mathrm{BM}$, including leptomeningeal metastases (LM), as long as they were stable and asymptomatic, thus mirroring what is clinically observed in real-life. On the other side, baseline brain scans were needed only for patients with suspected/ known CNS lesions (or up to local staging preferences), and this could have underestimated the actual incidence of CNS involvement in this study population by leaving out asymptomatic BM.

In the analysis performed by Reungwetwattana, the median CNS PFS was not reached in patients who received osimertinib versus a first-generation EGFR inhibitor, and the data favored osimertinib (95\% CI, 16.5 months-NR) over 13.9 months (95\% CI, 8.3 months-NR) of standard TKIs (HR, 0.48; 95\% CI, 0.26-0.86, $\mathrm{P}=0.014$ ), after a 
median follow-up of 12.4 months. Even more interestingly, the vast majority of CNS progressions were driven by the onset of new BM rather than progression of preexisting ones in both the arms, and less patients in the osimertinib arm developed new BM compared to the control arm (12\% versus $30 \%)$. This clearly indicates that osimertinib was decisive in preventing the appearance of new BMs in patients with known CNS lesions. Osimertinib strikingly achieved a CNS ORR of $91 \%$ in the subgroup of patients with $\geq 1$ measurable $\mathrm{BM}$, that largely exceeded the $68 \%$ obtained with standard TKI, even if not reaching the statistical significance [odds ratio (OR), 4.6; 95\% CI, 0.934.9, $\mathrm{P}=0.066$, and the benefit was observed regardless of previous brain irradiation (16). Osimertinib disease control rate hit $95 \%$ in this population (95\% CI, 77-100\%) versus $89 \%$ with standard TKI (95\% CI, 67-99\%), but this was also not statistically significant (OR, 2.5; 95\% CI, 0.2-55.8, $\mathrm{P}=0.462$ ), mainly due to the very small number of patients. In addition, looking at the five patients with suspected LM in the osimertinib group, complete responses were surprisingly documented in four cases (80\%) (16).

When patients with non-measurable BM were included in the analysis, osimertinib CNS ORR decreased to $66 \%$, but this percentage was significantly higher than the $43 \%$ reached in the control arm (OR, 2.5; 95\% CI, 1.2-5.2, $\mathrm{P}=0.011)$ and concordant with previous reports $(14,15)$. Intracranial responses obtained with osimertinib were also matched with extra-cranial tumor shrinkage $(77 \%$ of concordance), suggesting that the drug successfully acts in both the districts (16).

The results showed by Reungwetwattana further confirmed osimertinib activity in BM from EGFR-mutated NSCLC, following AURA (14), AURA2 (14) and AURA3 (15) results. Osimertinib more than doubled the ORR and PFS in CNS lesions previously met with platinum-based chemotherapy (24), and almost overtook the historical outcomes of highly effective stereotactic brain radiotherapy in this setting (25). The protective role of osimertinib on the development of new CNS lesions, already documented among the overall population of FLAURA trial (19), was also settled in patients with known BM at baseline, and osimertinib demonstrated an encouraging activity on LM. Of note, it should be pointed out that in this analysis gefitinib/erlotinib underperformed in CNS, in terms of CNS ORR, compared to previous findings (9-12) (Table 1), even though a direct comparison between the results could not be performed, due to the disparate designs of the studies and different sample sizes (24). In addition, median baseline CNS target lesion's size was greater in the control arm $(29 \mathrm{~mm})$ than osimertinib arm $(16 \mathrm{~mm})$, and this could have disadvantaged gefitinib/erlotinib (16).

As formerly approached, the lack of a systematic brain evaluation at study entry is worth to be recalled as a limitation to data interpretation. In the "parallel" ALEX trial comparing first-line alectinib versus crizotinib in $A L K$ positive patients, the baseline availability of CNS study makes the results more robust $(26,27)$. Additional limitations of this analysis include the lack of statistical significance of part of the outcomes, the relative small number of patients, little imbalances on patients' characteristics between the two arms, the lack of radiological assessment performed according to Response Assessment in Neuro-Oncology (RANO)-Brain Metastases (BM) criteria and the absence of an afatinib arm (which was still not superior to firstgeneration TKI in CNS disease) $(6,13,28)$. However, these findings certainly add consistent value to the use of osimertinib for the first-line treatment of EGFR-mutated NSCLC. Indeed, the CNS ORR achieved by osimertinib in first-line is even higher than what observed after a first-/second-generation TKI $(14,15)$, conforming to the other clinical outcomes which were improved when the compound was administered beforehand (19). Even if median intracranial PFS was still not reached for frontline osimertinib (95\% CI, 16.5 months-NR), it appeared to be highly promising compared to osimertinib' performance after the failure of previous TKI (11.7 months, 95\% CI, 10.0 months-NC) (15). Mature CNS PFS data are largely awaited, especially if we consider that the lower $95 \%$ CI limit of CNS PFS documented in this analysis (16.5 months) is fairly close to the overall median PFS achieved by osimertinib in the FLAURA study (18.9 months), sensing that the privileged brain sanctuary could not likewise escape the wide osimertinib antitumor effect (19). Moreover, the benefits derived from osimertinib on brain involvement would not be limited exclusively to the fraction of patients (50-60\%) who acquired the T790M mutation after failure of previous TKI. This turns more relevant recalling that CNS-progressing cases require T790M detection in blood or tumor for osimertinib prescription, even if brain disease in less T790M-dependent (8).

Notwithstanding, a full comprehension of which is the better TKI strategy in EGFR-mutated NSCLC (osimertinib upfront versus sequential) is still far to be achieved, if we consider that solid data about outcomes of patients who crossed over from standard TKI towards osimertinib are lacking, especially regarding CNS activity 
of the compounds (18). Not least, we speculate that the protective role of osimertinib on CNS, documented in the subgroup of patients with known CNS lesions at baseline, could reflect into a similar benefit in the overall population, including patients with no history of BM. In conclusion, the results provided in the analysis by Reungwetwattana make osimertinib doubtless more effective than standard TKIs in penetrating and treating the CNS sanctuary site, and then design osimertinib as the current best therapeutic option for $E G F R$-driven NSCLC patients. Intracranial disease control and disease prevention exerted by front-line osimertinib may deeply impact on global OS benefit still guaranteeing good quality of life, the true goals for all patients.

\section{Acknowledgements}

Funding: This work was supported by AIRC (Italian Association for Cancer Research), Milan (grant No. IG2017-20074; PI: M Tiseo).

\section{Footnote}

Conflicts of Interest: M Tiseo: Advisory boards and speakers' fee for Astra-Zeneca. The other authors have no conflicts of interest to declare.

\section{References}

1. Lin JJ, Cardarella S, Lydon CA, et al. Five-Year Survival in EGFR-Mutant Metastatic Lung Adenocarcinoma Treated with EGFR-TKIs. J. Thorac Oncol 2016;11:556-65.

2. Peters S, Bexelius C, Munk V, et al. The impact of brain metastasis on quality of life, resource utilization and survival in patients with non-small-cell lung cancer. Cancer Treat Rev 2016;45:139-62.

3. Rangachari D, Yamaguchi N, VanderLaan PA, et al. Brain metastases in patients with EGFR-mutated or ALKrearranged non-small-cell lung cancers. Lung Cancer 2015;88:108-11.

4. Ge M, Zhuang Y, Zhou X, et al. High probability and frequency of EGFR mutations in non-small cell lung cancer with brain metastases. J Neurooncol 2017;135:413-8.

5. Togashi Y, Masago K, Masuda S, et al. Cerebrospinal fluid concentration of gefitinib and erlotinib in patients with non-small cell lung cancer. Cancer Chemother Pharmacol 2012;70:399-405.

6. Hoffknecht P, Tufman A, Wehler T, et al. Efficacy of the irreversible ErbB family blocker afatinib in epidermal growth factor receptor (EGFR) tyrosine kinase inhibitor (TKI)-pretreated non-small-cell lung cancer patients with brain metastases or leptomeningeal disease. J Thorac Oncol 2015;10:156-63.

7. Hata AN, Niederst MJ, Archibald HL, et al. Tumor cells can follow distinct evolutionary paths to become resistant to epidermal growth factor receptor inhibition. Nat Med 2016;22:262-9.

8. Hata A, Katakami N, Yoshioka H, et al. Spatiotemporal T790M Heterogeneity in Individual Patients with EGFRMutant Non-Small-Cell Lung Cancer after Acquired Resistance to EGFR-TKI. J Thorac Oncol 2015;10:1553-9.

9. Porta R, Sánchez-Torres JM, Paz-Ares L, et al. Brain metastases from lung cancer responding to erlotinib: The importance of EGFR mutation. Eur Respir J 2011;37:624-31.

10. Wu YL, Zhou C, Cheng Y, et al. Erlotinib as second-line treatment in patients with advanced non-small-cell lung cancer and asymptomatic brain metastases: a phase II study (CTONG-0803). Ann Oncol 2013;24:993-9.

11. Hotta K, Kiura K, Ueoka H, et al. Effect of gefitinib ("Iressa", ZD1839) on brain metastases in patients with advanced non-small-cell lung cancer. Lung Cancer 2004;46:255-61.

12. Iuchi T, Shingyoji M, Sakaida T, et al. Phase II trial of gefitinib alone without radiation therapy for Japanese patients with brain metastases from EGFR-mutant lung adenocarcinoma. Lung Cancer 2013;82:282-7.

13. Schuler M, Wu YL, Hirsh V, et al. First-Line Afatinib versus Chemotherapy in Patients with Non-Small Cell Lung Cancer and Common Epidermal Growth Factor Receptor Gene Mutations and Brain Metastases. J Thorac Oncol 2016;11:380-90.

14. Goss G, Tsai CM, Shepherd FA, et al. CNS response to osimertinib in patients with T790M-positive advanced NSCLC: pooled data from two phase II trials. Ann Oncol 2018;29:687-93.

15. Wu YL, Ahn MJ, Garassino MC, et al. CNS Efficacy of Osimertinib in Patients With T790M-Positive Advanced Non-Small-Cell Lung Cancer: Data From a Randomized Phase III Trial (AURA3). J Clin Oncol 2018;36:2702-9.

16. Reungwetwattana T, Nakagawa K, Cho BC, et al. CNS Response to Osimertinib Versus Standard Epidermal Growth Factor Receptor Tyrosine Kinase Inhibitors in Patients With Untreated EGFR-Mutated Advanced Non-Small-Cell Lung Cancer. J Clin Oncol 2018:JCO2018783118. [Epub ahead of print].

17. Mok TS, Wu YL, Ahn MJ, et al. Osimertinib or PlatinumPemetrexed in EGFR T790M-Positive Lung Cancer. N 
Engl J Med 2017;376:629-40.

18. Recondo G, Facchinetti F, Olaussen KA, et al. Making the first move in EGFR-driven or ALK-driven NSCLC: firstgeneration or next-generation TKI? Nat Rev Clin Oncol 2018;15:694-708.

19. Soria JC, Ohe Y, Vansteenkiste J, et al. Osimertinib in Untreated EGFR-Mutated Advanced Non-Small-Cell Lung Cancer. N Engl J Med 2018;378:113-25.

20. Approved Drugs - Osimertinib (TAGRISSO). Available online: https://www.fda.gov/drugs/informationondrugs/ approveddrugs/ucm549683.htm

21. Tagrisso receives full approval in the EU. Available online: https://www.astrazeneca.com/media-centre/pressreleases/2017/tagrisso-receives-full-approval-in-theeu-25042017.html

22. Ballard P, Yates JW, Yang Z, et al. Preclinical Comparison of Osimertinib with Other EGFR-TKIs in EGFR-Mutant NSCLC Brain Metastases Models, and Early Evidence of Clinical Brain Metastases Activity. Clin Cancer Res 2016;22:5130-40.

23. Colclough N, Ballard PG, Barton P, et al. Preclinical comparison of the blood brain barrier (BBB) permeability

Cite this article as: Leonetti A, Facchinetti F, Tiseo M. Upfront osimertinib in EGFR-mutated non-small cell lung cancer: is brain still a sanctuary? Ann Transl Med 2018;6(Suppl 2):S110. doi: 10.21037/atm.2018.11.69 of osimertinib (AZD9291) with other irreversible next generation EGFR TKIs. Eur J Cancer 2016;69:S28.

24. Remon J, Besse B. Brain Metastases in Oncogene-Addicted Non-Small Cell Lung Cancer Patients: Incidence and Treatment. Front Oncol 2018;8:88.

25. Soon YY, Leong CN, Koh WY, et al. EGFR tyrosine kinase inhibitors versus cranial radiation therapy for EGFR mutant non-small cell lung cancer with brain metastases: a systematic review and meta-analysis. Radiother Oncol 2015;114:167-72.

26. Peters S, Camidge DR, Shaw AT, et al. Alectinib versus Crizotinib in Untreated ALK -Positive Non-Small-Cell Lung Cancer. N Engl J Med 2017;377:829-38.

27. Thai AA, Solomon BJ. Alectinib as first-line therapy for ALK-rearranged NSCLC: A no-brainer? Ann Oncol 2018;29:2160-2.

28. Park K, Tan EH, O'Byrne K, et al. Afatinib versus gefitinib as first-line treatment of patients with EGFR mutationpositive non-small-cell lung cancer (LUX-Lung 7): a phase 2B, open-label, randomised controlled trial. Lancet Oncol 2016;17:577-89. 